CERN-TH/96-282

hep-ph/9610471

\title{
Exploring the Invisible Renormalon: Renormalization of the Heavy-Quark Kinetic Energy
}

\author{
Matthias Neubert \\ Theory Division, CERN, CH-1211 Geneva 23, Switzerland
}

\begin{abstract}
Using the virial theorem of the heavy-quark effective theory, we show that the mixing of the operator for the heavy-quark kinetic energy with the identity operator is forbidden at the one-loop order by Lorentz invariance. This explains why such a mixing was not observed in several one-loop calculations using regularization schemes with a Lorentz-invariant UV regulator, and why no UV renormalon singularity was found in the matrix elements of the kinetic operator in the bubble approximation (the "invisible renormalon"). On the other hand, we show that the mixing is not protected in general by any symmetry, and it indeed occurs at the two-loop order. This implies that the parameter $\lambda_{1}^{H}$ of the heavy-quark effective theory is not directly a physical quantity, but requires a non-perturbative subtraction.
\end{abstract}

(Submitted to Physics Letters B)

CERN-TH/96-282

October 1996 


\section{Introduction}

The physics of hadrons containing a heavy quark simplifies greatly in the limit where the heavy-quark mass $m_{Q}$ is taken to infinity. Then new symmetries of the strong interactions arise, which relate the long-distance properties of many observables to a small number of hadronic matrix elements [1]. A systematic expansion around the heavy-quark limit is provided by the heavy-quark effective theory (HQET), in which a heavy quark inside a hadron moving with four-velocity $v$ is described by a velocity-dependent field $h_{v}(x)$ subject to the constraint $\psi h_{v}=$ $h_{v}$. The field $h_{v}(x)$ is related to the original heavy-quark field $Q(x)$ by a phase redefinition, which removes the large "mechanical" part $m_{Q} v$ of the heavy-quark momentum arising from the motion of the heavy hadron. Thus, the effective field carries the "residual momentum" $k=p_{Q}-m_{Q} v$, which characterizes the interactions of the heavy quark with gluons. The effective Lagrangian of the HQET is [2]- [4]

$$
\mathcal{L}_{\mathrm{eff}}=\bar{h}_{v} i v \cdot D h_{v}+\frac{1}{2 m_{Q}} \bar{h}_{v}\left(i D_{\perp}\right)^{2} h_{v}+\frac{C_{\mathrm{mag}} g_{s}}{4 m_{Q}} \bar{h}_{v} \sigma_{\mu \nu} G^{\mu \nu} h_{v}+O\left(1 / m_{Q}^{2}\right),
$$

where $D^{\mu}=\partial^{\mu}-i g_{s} A^{\mu}$ is the gauge-covariant derivative, and $D_{\perp}^{\mu}=D^{\mu}-$ $(v \cdot D) v^{\mu}$ contains its components orthogonal to the velocity. The origin of the operators arising at order $1 / m_{Q}$ is most transparent in the rest frame of the heavy hadron: the first operator corresponds to the kinetic energy resulting from the motion of the heavy quark inside the hadron (in the rest frame, $\left(i D_{\perp}\right)^{2}$ is the operator for $-\mathbf{k}^{2}$ ), whereas the second operator describes the magnetic interaction of the heavy-quark spin with the gluon field. The Wilson coefficient $C_{\text {mag }}$ results from short-distance effects and depends logarithmically on the scale at which the chromo-magnetic operator is renormalized. As a consequence of the so-called reparametrization invariance of the HQET (an invariance under infinitesimal changes of the velocity), the kinetic operator is not multiplicatively renormalized [5, 6].

The matrix elements of the two dimension-five operators in (11) play a most significant role in many applications of the HQET. They appear, for instance, in the spectroscopy of heavy hadrons and in the description of inclusive weak decays [1]. For the hadronic matrix elements of the kinetic operator, in particular, one defines hadronic parameters $\lambda_{1}^{H}$ by [7]

$$
\left\langle H(v)\left|\bar{h}_{v}\left(i D_{\perp}\right)^{2} h_{v}\right| H(v)\right\rangle=\lambda_{1}^{H}
$$

where we use a mass-independent normalization of states such that $\left\langle H(v)\left|\bar{h}_{v} h_{v}\right| H(v)\right\rangle=$ 1. Spectroscopic relations may be used to extract the difference in the values of $\lambda_{1}^{H}$ for two hadron states. For the ground-state heavy mesons and baryons, for

\footnotetext{
${ }^{1}$ Another common notation is to define $\mu_{\pi, H}^{2}=-\lambda_{1}^{H}$.
} 
instance, we obtain

$$
\frac{m_{\Lambda_{b}}-m_{\Lambda_{c}}}{\bar{m}_{B}-\bar{m}_{D}}=1+\frac{\lambda_{1}^{\mathrm{bar}}-\lambda_{1}^{\mathrm{mes}}}{2 \bar{m}_{D} \bar{m}_{B}}+O\left(1 / m_{H}^{3}\right),
$$

where $O\left(1 / m_{H}^{3}\right)$ means terms suppressed by three powers of the large hadron masses, and $\bar{m}_{B}=\frac{1}{4}\left(m_{B}+3 m_{B^{*}}\right)$ and $\bar{m}_{D}=\frac{1}{4}\left(m_{D}+3 m_{D^{*}}\right)$ denote the spinaveraged meson masses, defined such that they do not receive a contribution from the chromo-magnetic interaction. The value of a single parameter $\lambda_{1}^{H}$ cannot be determined from spectroscopy, since it always appears in combination with the heavy-quark mass, which by itself is not a physical parameter. This observation poses the questions about the status of the heavy-quark kinetic energy as a physical parameter.

In this letter, we study the mixing of the kinetic operator $\bar{h}_{v}\left(i D_{\perp}\right)^{2} h_{v}$ with the "identity operator" $\bar{h}_{v} h_{v}$ under ultraviolet (UV) renormalization. By naive dimensional analysis, such a mixing is expected to occur in regularization schemes with a dimensionful cutoff parameter $\lambda$, since the two operators have the same quantum numbers but different dimension. This would have important implications for phenomenology, as it leads to an additive contribution to the parameter $\lambda_{1}^{H}$ of the form $\lambda^{2} C\left[\alpha_{s}(\lambda)\right]$, which must be subtracted in order to define a renormalized ("physical") parameter that is independent of the UV regulator. The coefficient $C\left[\alpha_{s}(\lambda)\right]$ can be calculated order by order in an expansion in the small coupling constant $\alpha_{s}(\lambda)$, and it appears at first sight that the quadratically divergent term could be subtracted using perturbation theory. This impression is erroneous, however, because $C$ may contain non-perturbative contributions of the form $\exp \left[-8 \pi / \beta_{0} \alpha_{s}(\lambda)\right]=\left(\Lambda_{\mathrm{QCD}} / \lambda\right)^{2}$, which cannot be controlled in perturbation theory [8]. Such terms can contribute an amount of order $\Lambda_{\mathrm{QCD}}^{2}$ to the parameter $\lambda_{1}^{H}$, which is of the same order as the renormalized parameter itself. Hence, if the kinetic operator mixes with the identity operator, it is necessary that the quadratically divergent contribution to $\lambda_{1}^{H}$ be subtracted in a non-perturbative way, and hence the heavy-quark kinetic energy by itself is not directly a physical quantity. On the other hand, since the quadratic divergence is an UV effect and therefore insensitive to the nature of the external states, it follows that the difference in the values of $\lambda_{1}^{H}$ for two hadrons is a physical quantity, and relations such as (3) are meaningful.

The issue of power divergences of matrix elements is intimately related to that of UV renormalons [9]. The purpose of the heavy-quark expansion is to disentangle the short-distance physics characterized by the large mass scale $m_{Q}$ from the long-distance physics characterized by the typical scale of the momenta exchanged between the heavy quark and light degrees of freedom. This is achieved by introducing a factorization scale $\lambda$ such that $\Lambda_{\mathrm{QCD}} \ll \lambda \ll m_{Q}$. Contributions from momenta above $\lambda$ are controllable in perturbation theory and attributed to Wilson coefficients, whereas contributions from momenta below $\lambda$ are contained in the matrix elements of the operators in the HQET. If this program is performed 
with a "hard" factorization scale, these matrix elements diverge, for dimensional reasons, as powers of the UV cutoff. For practical reasons, however, one usually calculates the Wilson coefficients using dimensional regularization. In this case power divergences do not appear, and the factorization scale $\lambda$ is replaced by a renormalization scale $\mu$. It is then unavoidable that the Wilson coefficients receive contributions from momenta below $\mu$ (so-called infrared (IR) renormalons), and the operator matrix elements receive contributions from momenta above $\mu$ (socalled UV renormalons). These contributions lead to a factorial growth of the coefficients in the perturbative expansion of the Wilson coefficients and matrix elements. The corresponding perturbation series are divergent and not Borel summable; they must be truncated close to the minimal term. Therefore, the presence of renormalons leads to intrinsic ambiguities in the definition of the Wilson coefficients and matrix elements, which only cancel when all short- and long-distance contributions are combined in the heavy-quark expansion [10]-[16]. It is generally believed that from the degree of divergence of the matrix elements of an operator in the HQET one can deduce the position of the corresponding UV renormalon singularities in the Borel transform of these matrix elements with respect to the inverse coupling constant; a power divergence proportional to $\lambda^{n}$ corresponds to a singularity at the position $u=n / 2$ (in a certain normalization [15]) on the positive real axis in the Borel plane. For the case of the self-energy of a heavy quark, for instance, the correspondence between a linear UV divergence and a renormalon pole at $u=\frac{1}{2}$ has been established in 12, 13.

The question whether there is a mixing of the kinetic energy with the identity operator, and whether there exists a corresponding renormalon singularity, has been addressed previously by several authors, with seemingly controversial conclusions. At the one-loop order, such a mixing has indeed been observed when the HQET regularized on a space-time lattice [8]. Likewise, a "physical" definition of a parameter $\lambda_{1}^{H}(\lambda)$ has been suggested, which absorbs certain $O\left(\alpha_{s}\right)$ corrections appearing in the zero-recoil sum rules for heavy-quark transitions [17]. This definition is such that $\mathrm{d} \lambda_{1}^{H}(\lambda) / \mathrm{d} \lambda^{2} \propto \alpha_{s}(\lambda)$, indicating again a one-loop mixing of the kinetic energy with the identity operator. On the other hand, this mixing has not been observed at the one-loop order in two Lorentz-invariant cutoff regularization schemes [18], which use a Pauli-Villars regulator or a cutoff on the virtuality of the gluon in one-loop Feynman diagrams [19]. This observation appeared as a puzzle, because there seemed to be no obvious reason why the mixing should not occur at the one-loop order, and indeed individual diagrams were quadratically divergent. Yet, the vanishing of the operator mixing at the one-loop order has found its counterpart in the analysis of UV renormalons. As mentioned above, a quadratic divergence would correspond to a renormalon singularity at $u=1$ in the Borel plane. Even though we are far from being able to derive an exact expression for the Borel transform of a perturbation series, explicit results can be obtained in the so-called "bubble approximation", in which all terms corresponding to an arbitrary number of self-energy insertions on a gluon propagator 
are kept. Surprisingly, it was found that in this approximation the renormalon singularity at $u=1$ in the matrix elements of the kinetic operator is absent [18]. The absence of the related IR renormalon in the pole mass of a heavy quark was noted previously in [12]. This "missing" singularity has been called the "invisible renormalon". Three possible explanations for this puzzle have been suggested:

1. The vanishing of the operator mixing at the one-loop order is accidental and happens only in some peculiar regularization schemes. However, this does not explain why the renormalon singularity at $u=1$ is "invisible" in the bubble approximation.

2. The vanishing of the mixing is an artefact of one-loop perturbation theory. The mixing appears in higher orders, and the corresponding renormalon singularity appears when one goes beyond the bubble approximation.

3. There is a symmetry which prevents the mixing between the kinetic energy and the identity operator, but this symmetry is broken by the lattice regularization. Candidates of such symmetries are Lorentz (or rotational) invariance and the reparametrization invariance of the HQET [5, 6]. In fact, it has been conjectured that Lorentz invariance may protect the matrix elements of the kinetic energy against an UV renormalon singularity at $u=1$ [14]. However, later it was argued that there is no obvious connection between Lorentz or reparametrization invariance and the absence of a quadratic divergence (or the corresponding UV renormalon) in the matrix elements of the kinetic energy [18].

In this paper we shall resolve this puzzle. We will prove that the mixing between the kinetic energy and the identity operator is forbidden at the oneloop order in all regularization schemes with a Lorentz-invariant UV cutoff. For our arguments to hold, it is essential that the regulator be introduced in a fully Lorentz-invariant way; in particular, it is not sufficient to choose a regularization scheme that respects only rotational invariance in the rest frame of the heavy hadron. The fact that a mixing at the one-loop order was observed in \& 8 and [17] is a consequence of the explicit breaking of Lorentz invariance by the regularization schemes adopted in these calculations. On the other hand, in general there is no symmetry that protects the matrix elements of the kinetic operator from quadratic divergences, and we show explicitly that a mixing with the identity operator occurs from the two-loop order on. In the renormalon language, this suggest that there is a singularity at $u=1$ in the Borel plane, which would appear when one goes beyond the bubble approximation. Hence, each of the above-mentioned suggestions is only partly correct, and the puzzle of the "invisible renormalon" finds its explanation in an "accidental" space-time symmetry, which is only operative at the one-loop order. 

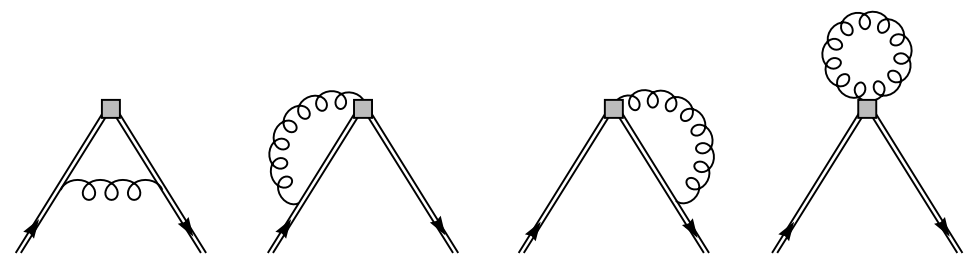

Figure 1: One-loop diagrams that could contribute to the mixing of the kinetic energy with the identity operator. The kinetic operator is represented by a grey square; heavy-quark propagators are drawn as double lines.

\section{Operator Mixing}

As mentioned above, in regularization schemes with a dimensionful cutoff $\lambda$, the kinetic operator can mix with the identity operator under UV renormalization. We define a renormalization constant $Z_{D^{2} \rightarrow \hat{1}}$ by

$$
\left.\bar{h}_{v}\left(i D_{\perp}\right)^{2} h_{v}\right|_{\text {bare }}=Z_{D^{2} \rightarrow \hat{1}} \bar{h}_{v} h_{v}+\ldots
$$

where the subscript "bare" indicates the bare operator, whose matrix elements depend on the UV regulator, and the ellipses represent contributions from operators of higher dimension. Such a mixing leads to an additive contribution to the matrix elements of the kinetic operator which, for dimensional reasons, is quadratically divergent in the cutoff $\lambda$. It follows that

$$
\frac{\mathrm{d}}{\mathrm{d} \lambda^{2}} \lambda_{1}^{H}(\lambda)=\frac{\mathrm{d}}{\mathrm{d} \lambda^{2}} Z_{D^{2} \rightarrow \hat{1}}
$$

Our goal is to perform a perturbative calculation of $Z_{D^{2} \rightarrow \hat{1}}$ in a regularization scheme where the cutoff preserves Lorentz invariance. By evaluating the UV divergences of the diagrams shown in Fig. 11 (supplemented by the wave-function renormalization of the quark fields), it has been shown in [18] that $Z_{D^{2} \rightarrow \hat{1}}$ vanishes at the one-loop order in two such regularization schemes. However, individual diagrams have a quadratic divergence, and the reason for the cancellations occurring in the sum of all diagrams remained unclear.

To extend the direct calculation of $Z_{D^{2} \rightarrow \hat{1}}$ to the next order would involve the evaluation of a large number of two-loop diagrams. In this letter, we suggest a more efficient way to perform this calculation, which in addition will provide an explanation for the vanishing of $Z_{D^{2} \rightarrow \hat{1}}$ at the one-loop order. To this end, we use the virial theorem of the HQET, which establishes a relation between the kinetic energy and a matrix element of an operator containing the gluon field-strength tensor between states moving at different velocities [7, 20]. For the ground-state 

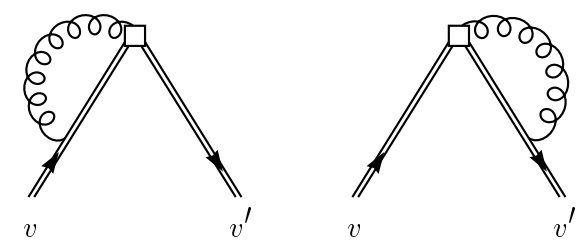

Figure 2: One-loop diagrams that could contribute to the mixing of the chromoelectric operator with lower-dimensional operators. The velocity-changing chromo-electric operator is represented by a white square.

pseudoscalar mesons and $\Lambda_{Q}$ baryons, the virial theorem states that

$$
\left\langle H\left(v^{\prime}\right)\left|\bar{h}_{v^{\prime}} i g_{s} G^{\mu \nu} h_{v}\right| H(v)\right\rangle=\left(v^{\mu} v^{\prime \nu}-v^{\nu} v^{\prime \mu}\right)\left\{-\frac{\lambda_{1}^{H}}{3}+O\left(v \cdot v^{\prime}-1\right)\right\} .
$$

This is a non-perturbative relation between hadronic matrix elements, which is preserved by renormalization [21]. In the rest frame of one of the hadrons, only the electric field has a non-vanishing matrix element, and we shall therefore refer to the operator on the left-hand side as the chromo-electric operator. Note that (6) is not an operator identity, but rather a relation between ground-state matrix elements; the corresponding operator identity would contain operators of higher spin, which have vanishing ground-state matrix elements. However, (6) is an operator identity as far as the quadratic UV divergences are concerned.

Similar to the kinetic operator, also the chromo-electric operator can mix with a dimension-three operator. Since the Feynman rules of the HQET do not involve Dirac matrices, there is only one candidate for such an operator, and we define

$$
\left.\bar{h}_{v^{\prime}} i g_{s} G^{\mu \nu} h_{v}\right|_{\text {bare }}=Z_{E \rightarrow \hat{1}}\left(v \cdot v^{\prime}\right)\left(v^{\mu} v^{\prime \nu}-v^{\nu} v^{\prime \mu}\right) \bar{h}_{v^{\prime}} h_{v}+\ldots .
$$

Although there exist other dimension-three operators with the right quantum numbers, such as $\bar{h}_{v^{\prime}} i \sigma^{\mu \nu} h_{v}$ and $\bar{h}_{v^{\prime}}\left[\left(v-v^{\prime}\right)^{\mu} \gamma^{\nu}-\left(v-v^{\prime}\right)^{\nu} \gamma^{\mu}\right] h_{v}$, they cannot be induced by quantum effects. The virial theorem (6) implies a relation between the renormalization factors $Z_{E \rightarrow \hat{1}}$ and $Z_{D^{2} \rightarrow \hat{1}}$ in the limit of equal velocities $\left(v \cdot v^{\prime}=1\right)$, which reads

$$
Z_{D^{2} \rightarrow \hat{1}}=-3 Z_{E \rightarrow \hat{1}}(1)
$$

Thus, if we understand the mixing of the chromo-electric operator in (7), we also understand the mixing of the kinetic energy with the identity operator. As we shall see, calculating $Z_{D^{2} \rightarrow \hat{1}}$ by means of the relation (8) is much more efficient than a direct calculation.

Since the gluon field-strength is an antisymmetric Lorentz tensor, the only possible dimension-three operator appearing on the right-hand side of (7) contains

\footnotetext{
${ }^{2}$ The same relation holds for the ground-state vector mesons, when an averaging over the two transverse polarization states is implied.
} 
both velocities, $v$ and $v^{\prime}$. Consider now the diagrams shown in Fig. 2, which could potentially give rise to operator mixing at the one-loop order. Let us assume that we regulate the UV divergences of these diagrams in a Lorentz-invariant way, for instance, by introducing a Pauli-Villars cutoff. Since one leg of the gluon must be attached to the chromo-electric operator, there is no way to obtain a dependence on both heavy-quark velocities; the first diagram can only depend on $v$, while the second one can only depend on $v^{\prime}$. Hence, because of Lorentz invariance the renormalization constant $Z_{E \rightarrow \hat{1}}$ must vanish at the one-loop order, and so does $Z_{D^{2} \rightarrow \hat{1}}$. The relation (8) implied by the virial theorem thus provides for a very simple explanation of the apparently accidental cancellations between the quadratic divergences of the one-loop diagrams in Fig. 1, observed in [18].

On the other hand, the above argument is clearly linked to the one-loop order. Let us, therefore, study the problem at the level of two loops. Then there are four diagrams that could potentially lead to a dependence on both heavy-quark velocities. They are shown in Fig. 3. (Notice that this is a vast simplification over the direct calculation of the two-loop mixing of the kinetic operator.) We need to calculate the UV divergences of these diagrams in a regularization scheme with a dimensionful cutoff. However, we have to be careful not to spoil Lorentz or gauge invariance when introducing the cutoff. For the case at hand, this can be done using dispersion relations. Let us consider the matrix element of the chromoelectric operator evaluated between quark states with residual momenta $k$ and $k^{\prime}$. (The nature of the external states is irrelevant, since we are only interested in the UV behaviour of the diagrams.) This matrix element has discontinuities if either $v \cdot k$ or $v^{\prime} \cdot k^{\prime}$ are positive, since then physical intermediate states can be excited. The Feynman amplitudes corresponding to the diagrams in Fig. 3 satisfy a dispersion representation of the form ${ }^{3}$

$$
A=\int \mathrm{d} \omega \int \mathrm{d} \omega^{\prime} \frac{\rho\left(\omega, \omega^{\prime}\right)}{(\omega-v \cdot k-i \epsilon)\left(\omega^{\prime}-v^{\prime} \cdot k^{\prime}-i \epsilon\right)}
$$

where $\rho\left(\omega, \omega^{\prime}\right)$ is the double spectral density. The variables $\omega$ and $\omega^{\prime}$ have a concrete physical meaning as the energies of the intermediate states, measured with respect to the heavy-quark mass. A mixing with the identity operator would show up as a quadratic divergence of the dispersion integral arising from the region of large energies. Introducing an UV cutoff in the dispersion integral regulates these divergences without spoiling any of the symmetries of the theory; in particular, Lorentz and gauge invariance are preserved.

Below, we shall investigate two different prescriptions how to regulate the dispersion integral. The most direct way is to introduce a hard UV cutoff, i.e.

$$
A^{\mathrm{reg}}=\int^{\lambda} \mathrm{d} \omega \int^{\lambda} \mathrm{d} \omega^{\prime} \frac{\rho\left(\omega, \omega^{\prime}\right)}{\omega \omega^{\prime}} .
$$

\footnotetext{
${ }^{3}$ Once the double integral is regulated in the UV region, there are no subtraction terms in the dispersion relation.
} 

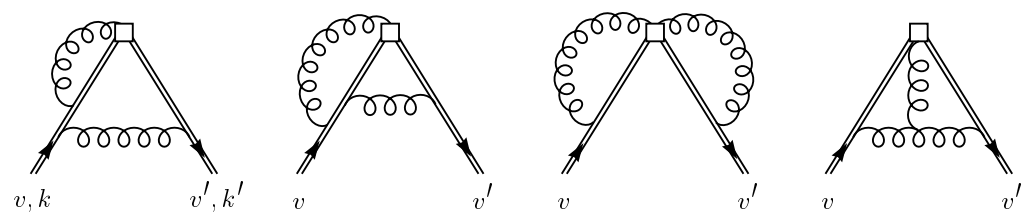

Figure 3: Two-loop diagrams that could contribute to the mixing of the chromoelectric operator with lower-dimensional operators. Not shown are two copies of the first two diagrams with the "outer" gluon attached to the other heavy-quark line.

As we are interested in studying the UV behaviour of the operator matrix element, we have set the external momenta to zero (there are no IR divergences). For dimensional reasons, the result will then be quadratic in the cutoff $\lambda$. Another scheme is to perform a double Borel transformation of the original integral in the external variables $v \cdot k$ and $v^{\prime} \cdot k^{\prime}$. This leads to

$$
A^{\text {Borel }}=\frac{1}{M M^{\prime}} \int \mathrm{d} \omega \int \mathrm{d} \omega^{\prime} \rho\left(\omega, \omega^{\prime}\right) e^{-\omega / M-\omega^{\prime} / M^{\prime}},
$$

corresponding to a "soft" exponential cutoff. It is most natural to set the Borel parameters equal: $M=M^{\prime}=\lambda$, in which case the result will be proportional to $\lambda^{2}$.

To evaluate the two-loop diagrams in Fig. 3, we work in the Feynman gauge; the sum of all diagrams is gauge independent. Using the Feynman rules of the HQET, it is then straightforward to see that the first two diagrams vanish. The third diagram is easily calculated, since it factorizes into two one-loop integrals. Using dimensional regularization, with $d$ being the number of space-time dimensions, we obtain

$$
\begin{aligned}
& -2 C_{F} C_{A} \frac{g_{s}^{4}}{(4 \pi)^{d}} \Gamma^{2}(3-d) \Gamma^{2}(d / 2-1)(-2 v \cdot k)^{d-3}\left(-2 v^{\prime} \cdot k^{\prime}\right)^{d-3} \\
& \quad \times\left(v^{\mu} v^{\prime \nu}-v^{\nu} v^{\prime \mu}\right) \bar{u}_{v^{\prime}}\left(k^{\prime}\right) u_{v}(k) .
\end{aligned}
$$

Here $C_{F}=\frac{1}{2}\left(N_{c}^{2}-1\right) / N_{c}$ and $C_{A}=N_{c}$ are the eigenvalues of the quadratic Casimir operator in the fundamental and the adjoint representations, and $u_{v}(k)$ is a heavy-quark spinor. The external momenta $k$ and $k^{\prime}$ act as IR regulators. The result is real if $v \cdot k<0$ and $v^{\prime} \cdot k^{\prime}<0$. However, as expected there are discontinuities for positive values of these variables. The corresponding double spectral density is finite in four dimensions and given by

$$
-C_{F} C_{A} \frac{\alpha_{s}^{2}}{2 \pi^{2}} \theta(\omega) \theta\left(\omega^{\prime}\right) \omega \omega^{\prime}\left(v^{\mu} v^{\prime \nu}-v^{\nu} v^{\prime \mu}\right) \bar{u}_{v^{\prime}}\left(k^{\prime}\right) u_{v}(k)
$$

The calculation of the last diagram is more involved, since this is a genuine twoloop diagram with a non-trivial dependence on the variable $v \cdot v^{\prime}$. We shall first 
discuss the result obtained in the scheme based on using a double Borel transformation. For this case, efficient techniques to calculate two-loop integrals involving two heavy quarks moving at different velocities have been developed in [22]. In the case of equal Borel parameters $\left(M=M^{\prime}=\lambda\right)$ we find, after combining the results for the two diagrams, that the renormalization factor defined in (7) is given by

$$
Z_{E \rightarrow \hat{1}}^{\text {Borel }}\left(v \cdot v^{\prime}\right)=-C_{F} C_{A} \frac{\alpha_{s}^{2}}{4 \pi^{2}} F\left(v \cdot v^{\prime}\right) \lambda^{2},
$$

where

$$
F(w)=\frac{3 w+2}{\left(w^{2}-1\right)^{3 / 2}}\left[L_{2}\left(-w_{+}\right)-L_{2}\left(-w_{-}\right)\right]+\frac{2 w+3}{w^{2}-1} \ln [2(w+1)]-\frac{1}{w+1} .
$$

Here $w_{ \pm}=w \pm \sqrt{w^{2}-1}$, and $L_{2}(x)=-\int_{0}^{x} \frac{\mathrm{d} y}{y} \ln (1-y)$ is the dilogarithm. Evaluating this expression in the equal-velocity limit, using that $F(1)=\frac{1}{3}(1+$ $2 \ln 2$ ), we obtain from (8) the two-loop renormalization constant for the mixing of the kinetic energy with the identity operator. The result is

$$
Z_{D^{2} \rightarrow \hat{1}}^{\text {Borel }}=C_{F} C_{A} \frac{\alpha_{s}^{2}}{4 \pi^{2}}(1+2 \ln 2) \lambda^{2} .
$$

The precise numerical coefficient of the quadratic divergence depends on the way in which the UV cutoff $\lambda$ is introduced; below we will derive the result for another scheme. However, the fact that we have found a regularization scheme that preserves Lorentz and gauge invariance, and in which the kinetic energy mixes with the identity operator at the two-loop order, proves that such a mixing is present in general. Beyond the one-loop order, it is not forbidden by any symmetry.

Let us now elaborate on the result (16). We have calculated the generalization of this relation to the case of unequal Borel parameters. Then

$$
Z_{D^{2} \rightarrow \hat{1}}^{\text {Borel }}=C_{F} C_{A} \frac{\alpha_{s}^{2}}{4 \pi^{2}} M M^{\prime} G\left(M / M^{\prime}\right),
$$

where

$$
G(x)=\frac{2\left(2-3 x+2 x^{2}\right)}{x} \ln (1+x)+\frac{2 x^{2}\left(1-3 x-2 x^{2}\right)}{(1+x)^{3}} \ln x-\frac{1-6 x+x^{2}}{(1+x)^{2}} .
$$

The function $G(x)$ is symmetric under the exchange $x \leftrightarrow 1 / x$ and obeys $G(1)=$ $1+2 \ln 2$. Expression (17) is useful, since it allows us to derive the double spectral density in the dispersion integral (9). It is obtained by performing another double Borel transformation, now in the variables $-1 / M$ and $-1 / M^{\prime}$, with Borel parameters $1 / \omega$ and $1 / \omega^{\prime}$ [23]. The calculation is straightforward and leads to

$$
Z_{D^{2} \rightarrow \hat{1}}=C_{F} C_{A} \frac{\alpha_{s}^{2}}{4 \pi^{2}} \int \mathrm{d} \omega \int \mathrm{d} \omega^{\prime} \frac{\bar{\rho}\left(\omega, \omega^{\prime}\right)}{\omega \omega^{\prime}},
$$


where

$$
\bar{\rho}\left(\omega, \omega^{\prime}\right)=\theta(\omega) \theta\left(\omega^{\prime}\right)\left\{\omega \omega^{\prime}+5 \omega_{<}^{2}+2\left(3 \omega \omega^{\prime}-\omega^{2}-\omega^{\prime 2}\right) \ln \left(1-\frac{\omega_{<}}{\omega_{>}}\right)\right\},
$$

with $\omega_{<}=\min \left(\omega, \omega^{\prime}\right)$ and $\omega_{>}=\max \left(\omega, \omega^{\prime}\right)$. With the explicit result for the double spectral density at hand, we can now evaluate, according to (10), the renormalization factor in the scheme with a hard UV cutoff. The result is

$$
Z_{D^{2} \rightarrow \hat{1}}^{\mathrm{reg}}=C_{F} C_{A} \frac{\alpha_{s}^{2}}{4 \pi^{2}}\left(\frac{\pi^{2}}{3}-1\right) \lambda^{2} .
$$

As expected, the numerical coefficient in front of the quadratic divergence is different from the case of the Borel regulator in (16), but the general structure of the result is the same in both regularization schemes.

We emphasize that in deriving that there is no mixing at the one-loop order it is crucial that the UV cutoff be introduced in a fully Lorentz-invariant way; in particular, for our arguments to hold it is not sufficient to choose a regularization scheme that respects only rotational invariance in the rest frame of the heavy hadron. This is relevant when comparing our results to some previous calculations, in which a non-vanishing contribution to $Z_{D^{2} \rightarrow \hat{1}}$ was obtained at $O\left(\alpha_{s}\right)$. In the case of the lattice regularization, the one-loop mixing of the kinetic energy with the identity operator arises because the lattice breaks Lorentz invariance (i.e., Euclidean rotational invariance). The result for the renormalization factor $Z_{D^{2} \rightarrow \hat{1}}$ in this scheme is [8]

$$
Z_{D^{2} \rightarrow \hat{1}}^{\text {latt }}=-k_{\text {latt }} C_{F} \frac{\alpha_{s}}{\pi} \lambda^{2},
$$

where $\lambda=\pi / a$ is the UV cutoff ( $a$ is the lattice spacing), and the numerical coefficient $k_{\text {latt }}$ is given by

$$
k_{\text {latt }}=\frac{1}{2 \pi^{3}} \int_{-\pi}^{\pi} \mathrm{d}^{3} k \frac{1}{\sqrt{(1+A)^{2}-1}}, \quad A=\sum_{i=1}^{3}\left(1-\cos k_{i}\right) .
$$

The appearance of the quantity $A$ shows that the lattice regularization breaks both Lorentz and rotational invariance. Things are slightly more intricate with the scheme adopted in [17], however. The definition for the parameter $\lambda_{1}^{H}(\lambda)$ suggested there is equivalent to introducing a cutoff on the spatial momentum $(|\mathbf{k}|<\lambda)$ in the rest frame of the hadron containing the heavy quark. I Although working in the rest frame is quite natural when dealing with heavy quarks, this prescription breaks Lorentz invariance in that it specifies a particular reference frame, thereby introducing a dependence on the velocity $v$ through the regularization procedure. Then the arguments that led us to conclude that the one-loop

\footnotetext{
${ }^{4}$ The author is grateful to M. Shifman for pointing out this fact, and for suggesting the derivation of (26) along the lines discussed here.
} 
diagrams in Fig. 2 have no quadratic divergences are no longer valid. The second diagram, which explicitly depends on $v^{\prime}$, now acquires an implicit dependence on $v$ through the regularization, and thus it can contribute to $Z_{E \rightarrow \hat{1}}$ in (7). After a straightforward calculation, we find that

$$
Z_{E \rightarrow \hat{1}}\left(v \cdot v^{\prime}\right)=C_{F} \frac{\alpha_{s}}{2 \pi} H\left(v \cdot v^{\prime}\right) \lambda^{2}
$$

where

$$
H(w)=\frac{w-r(w)}{w^{2}-1}, \quad r(w)=\frac{1}{\sqrt{w^{2}-1}} \ln \left(w+\sqrt{w^{2}-1}\right) .
$$

The same result is obtained if the cutoff is introduced in the rest frame of the final-state hadron. Using that $H(1)=\frac{3}{2}$, we obtain from (8)

$$
Z_{D^{2} \rightarrow \hat{1}}=-C_{F} \frac{\alpha_{s}}{\pi} \lambda^{2}
$$

which agrees with the result derived in [17]. The same result can be recovered from (22) by taking the "continuum limit" $A \rightarrow \frac{1}{2} \mathbf{k}^{2}$ and imposing the cutoff on $|\mathbf{k}|$ (i.e. by integrating over a sphere rather than a cube), in which case rotational invariance is restored, and $k_{\text {latt }} \rightarrow 1$. Since the lattice version of the HQET is formulated for heavy quarks at rest, Lorentz invariance is broken even in the "continuum limit".

\section{Conclusions}

Using the virial theorem of the heavy-quark effective theory, we have shown that the mixing of the operator for the heavy-quark kinetic energy with the identity operator is forbidden at the one-loop order by Lorentz invariance. This resolves the puzzle of the "invisible renormalon" raised in [18], which consisted in the observation of an apparently accidental vanishing of the mixing at the one-loop order in several Lorentz-invariant cutoff regularization schemes, and the associated absence of an UV renormalon singularity in the bubble approximation. However, the mixing is not protected in general by any symmetry, and we have shown explicitly that it does occur at the two-loop order. This means that the parameter $\lambda_{1}^{H}$ of the heavy-quark effective theory is not directly a physical quantity, but requires a non-perturbative subtraction.

Our results for the two-loop renormalization of the kinetic energy imply that, in any regularization scheme with a dimensionful cutoff parameter $\lambda$ that preserves Lorentz and gauge invariance, there is an additive, quadratically divergent contribution to the parameter $\lambda_{1}^{H}(\lambda)$ of the HQET, such that

$$
\frac{\mathrm{d}}{\mathrm{d} \lambda^{2}} \lambda_{1}^{H}(\lambda)=\frac{\mathrm{d}}{\mathrm{d} \lambda^{2}} Z_{D^{2} \rightarrow \hat{1}}=k C_{F} C_{A} \frac{\alpha_{s}^{2}(\lambda)}{4 \pi^{2}}+O\left(\alpha_{s}^{3}\right) .
$$


For the two regularization schemes considered here, the numerical coefficient $k$ takes the values $(1+2 \ln 2) \approx 2.39$ and $\left(\frac{1}{3} \pi^{2}-1\right) \approx 2.29$. Note that this renormalization-group equation is different from the one derived in [17], which contains a one-loop contribution of the form $-C_{F} \alpha_{s}(\lambda) / \pi$ on the right-hand side, in accordance with (26). The reason is that the "physical" definition of the parameter $\mu_{\pi, H}^{2}(\lambda)=-\lambda_{1}^{H}(\lambda)$ suggested by these authors is equivalent to introducing a cutoff on the spatial momentum in the rest frame of the heavy hadron and thus breaks Lorentz invariance. (Although it is perfectly consistent, this definition may therefore not be the most convenient one.) The values of the kinetic energy defined in different regularization schemes can be related to each other using perturbation theory. From our results, it follows that at the one-loop order there is a universal relation between the parameter $\mu_{\pi, H}^{2}(\lambda)$ defined in [17] and $\lambda_{1}^{H}(\lambda)$ defined in any Lorentz-invariant regularization scheme, which reads

$$
\mu_{\pi, H}^{2}(\lambda)=-\lambda_{1}^{H}(\lambda)+C_{F} \frac{\alpha_{s}(\lambda)}{\pi} \lambda^{2}+O\left(\alpha_{s}^{2}\right) .
$$

This implies that the lower bound for the kinetic energy of a heavy quark inside a meson, $\mu_{\pi \text {,mes }}^{2}(\lambda)>0.36 \mathrm{GeV}^{2}$, which by construction holds for the definition proposed in [17], becomes weaker when the kinetic energy is regulated in a Lorentz-invariant way. Even if the cutoff $\lambda$ is chosen as low as $1 \mathrm{GeV}$, the extra term in (28) amounts to about $0.15 \mathrm{GeV}^{2}$, implying that the lower bound for $-\lambda_{1}^{\text {mes }}(\lambda)$ is at most $0.2 \mathrm{GeV}^{2}$. This observation is in accordance with arguments presented in [24], where a similar conclusion has been reached.

As mentioned in the introduction, the presence of a quadratic divergence entails that the parameter $\lambda_{1}^{H}$ of the HQET requires a non-perturbative subtraction to be defined and as such is not directly a physical parameter. Since in Lorentzinvariant regularization schemes the operator mixing occurs only at the nextto-leading order, one may hope that in practice the ambiguities related to the choice of the subtraction scheme will be small. Nevertheless, one has to be careful when comparing the values of $\lambda_{1}^{H}$ obtained using different theoretical methods. For instance, QCD sum-rule determinations of $\lambda_{1}^{H}$ for the ground-state heavy mesons [25] 27] and baryons [28] are performed in a scheme based on dimensional regularization, i.e. without a dimensionful UV cutoff. Strictly speaking, the results are then affected by a renormalon ambiguity problem, i.e. there is an intrinsic arbitrariness of order $\Lambda_{\mathrm{QCD}}^{2}$, which would show up when higher-order perturbative corrections to the sum rules would be calculated beyond the bubble approximation. Still, these results can consistently be used in connection with Wilson coefficients calculated to the same accuracy. Therefore, the sum-rule calculations may be compared with the results of phenomenological determinations of $\lambda_{1}^{\text {mes }}$ from a combined analysis of inclusive decay rates and moments of the decay spectra in decays of beauty and charm mesons [29]-31]. On the other hand, when $\lambda_{1}^{H}$ is determined using lattice gauge theory, the presence of a dimensionful regulator (the lattice spacing) requires a non-perturbative subtraction, and the 
results depend on how this subtraction is performed. A "physical prescription" for such a subtraction has been suggested in [32].

Acknowledgement: It is a pleasure to thank Martin Beneke, Chris Sachrajda and Misha Shifman for many useful discussions and suggestions.

\section{References}

[1] For a review, see: M. Neubert, Phys. Rep. 245, 259 (1994); Int. J. Mod. Phys. A 11, 4173 (1996).

[2] E. Eichten and B. Hill, Phys. Lett. B 243, 427 (1990).

[3] H. Georgi, Phys. Lett. B 240, 447 (1990).

[4] A.F. Falk, B. Grinstein and M.E. Luke, Nucl. Phys. B 357, 185 (1991).

[5] M. Luke and A.V. Manohar, Phys. Lett. B 286, 348 (1992).

[6] Y.-Q. Chen, Phys. Lett. B 317, 421 (1993).

[7] A.F. Falk and M. Neubert, Phys. Rev. D 47, 2965 and 2982 (1993).

[8] L. Maiani, G. Martinelli and C.T. Sachrajda, Nucl. Phys. B 368, 281 (1992).

[9] C.T. Sachrajda, in: Lattice 95, Nucl. Phys. B (Proc. Suppl.) 47, 100 (1996); G. Martinelli and C.T. Sachrajda, Phys. Lett. B 354, 423 (1995); preprint CERN-TH-96-117 (1996) hep-ph/9605336.

[10] F. David, Nucl. Phys. B 234, 237 (1984); 263, 637 (1986).

[11] A.H. Mueller, Nucl. Phys. B 250, 327 (1985).

[12] M. Beneke and V.M. Braun, Nucl. Phys. B 426, 301 (1994).

[13] I.I. Bigi, M.A. Shifman, N.G. Uraltsev and A.I. Vainshtein, Phys. Rev. D 50, 2234 (1994).

[14] M. Beneke, V.M. Braun and V.I. Zakharov, Phys. Rev. Lett. 73, 3058 (1994).

[15] M. Neubert and C.T. Sachrajda, Nucl. Phys. B 438, 235 (1995).

[16] M. Luke, A.V. Manohar and M.J. Savage, Phys. Rev. D 51, 4924 (1995).

[17] I. Bigi, M. Shifman, N.G. Uraltsev and A. Vainshtein, Phys. Rev. D 52, 196 (1995).

[18] G. Martinelli, M. Neubert and C.T. Sachrajda, Nucl. Phys. B 461, 238 (1996).

[19] M. Neubert, Phys. Rev. D 51, 5924 (1995).

[20] M. Neubert, Phys. Lett. B 322, 419 (1994).

[21] G. Amoros-Vincente and M. Neubert, in preparation. 
[22] M. Neubert, Phys. Rev. D 47, 4063 (1993).

[23] This technique has been first mentioned to the author by A. Radyushkin, and has been elaborated in: M. Neubert, Phys. Rev. D 46, 3914 (1992).

[24] A. Kapustin, Z. Ligeti, M.B. Wise and B. Grinstein, Phys. Lett. B 375, 327 (1996).

[25] V. Eletsky and E. Shuryak, Phys. Lett. B 276, 191 (1992).

[26] P. Ball and V.M. Braun, Phys. Rev. D 49, 2472 (1994).

[27] M. Neubert, preprint CERN-TH/96-208 (1996) [hep-ph/9608211, to appear in Phys. Lett. B.

[28] P. Colangelo, C.A. Dominguez, G. Nardulli and N. Paver, Phys. Rev. D 54, 4622 (1996).

[29] M. Gremm, A. Kapustin, Z. Ligeti and M.B. Wise, Phys. Rev. Lett. 77, 20 (1996).

[30] A.F. Falk, M. Luke and M.J. Savage, Phys. Rev. D 53, 6316 (1996).

[31] V. Chernyak, Novosibirsk preprint Budker INP-96-23 (1996) hepph/9604376.

[32] V. Giménez, G. Martinelli and C.T. Sachrajda, CERN preprint CERNTH/96-175 (1996) hep-lat/9607055. 